\title{
Benzodiazepine-Related Cognitive Impairment or Dementia: A Signal Detection Study Using a Case/Non-Case Approach
}

\author{
Yeon-Hee Baek ${ }^{1 *}$, Hye-Jun Kim ${ }^{1 *}$, Ji-Hwan Bae', Hyesung Lee ${ }^{1}$, \\ In-Sun Oh${ }^{1}$, Woo Jung Kim², and Ju-Young Shin ${ }^{1}$ \\ 1School of Pharmacy, Sungkyunkwan University, Suwon, Republic of Korea \\ ${ }^{2}$ Department of Psychiatry, Yongin Severance Hospital, Yonsei University College of Medicine, Yongin, Republic of Korea
}

Objective The association between benzodiazepine use and the risk of cognitive impairment or dementia has been controversial. Our study aims to detect this association through a case/non-case method using the Korea Institute of Drug Safety \& Risk Management-Korea adverse event reporting system database (KIDS-KD) between 2007 and 2016.

Methods Cases were adverse event (AE)-pairs with suspected cognitive impairment or dementia. 10 non-cases were matched to each case on age and sex. Exposure was defined as use of benzodiazepines, including long-, intermediate-, and short-acting benzodiazepine. We conducted multivariable logistic regression analyses to estimate reporting odds ratios (ROR) and 95\% confidence intervals (CI).

Results Of the 1,086,584 AE-pairs, 887 cases were suspected AE-pairs of cognitive impairment or dementia, and 775,444 non-cases were selected. Benzodiazepine use was associated with increased AE-pairs of cognitive impairment or dementia when assessed using those with certain, probable, and/or possible in causality assessments (ROR=2.69, 95\% CI=1.66-4.38). Higher ROR estimates were shown in female $(2.33,1.48-3.67)$ and in those with polypharmacy $(2.20,1.35-3.57)$. Dementia safety profiles were inconsistent across individual benzodiazepine components.

Conclusion These results suggest the potentially increased association between benzodiazepine use and cognitive impairment or dementia in female and those with polypharmacy. Inconsistent safety profiles of benzodiazepine components should be further investigated.

Psychiatry Investig 2020;17(6):587-595

Key Words Benzodiazepine, Dementia, Case/non-case, Signal detection, Korea Institute of Drug Safety \& Risk Management Korea Adverse Event Reporting System database (KIDS-KD).

\section{INTRODUCTION}

Benzodiazepines are among the most commonly prescribed sedative-hypnotics and are highly prevalent $(>10 \%)$ in elderly patients. ${ }^{1,2}$ However, many observational studies raised safety concerns over benzodiazepine use and the potential risk of dementia. ${ }^{3-5}$ Though the mechanism of the influence of benzodiazepine on the development of dementia is uncertain, there are hypotheses that, based on the neural compensation and

Received: October 14, 2019 Revised: February 20, 2020

Accepted: March 17, 2020

$\triangle$ Correspondence: Ju-Young Shin, PhD

School of Pharmacy, Sungkyunkwan University, 2066 Seobu-ro, Jangan-gu, Suwon 16419, Republic of Korea

Tel: +82-31-290-7702, Fax: +82-31-292-8800, E-mail: shin.jy@skku.edu

*These authors contributed equally to this work.

(c) This is an Open Access article distributed under the terms of the Creative Commons Attribution Non-Commercial License (https://creativecommons.org/licenses/by$\mathrm{nc} / 4.0$ ) which permits unrestricted non-commercial use, distribution, and reproduction in any medium, provided the original work is properly cited. cognitive reserve concepts, benzodiazepine users may utilize undamaged alternative neural networks to reduce cognitive disorders. ${ }^{6}$ However, by reducing the brain activation level, benzodiazepines could impair this ability and increase the potential risk of dementia. Susceptibility to benzodiazepine-induced dementia may differ across sex, ${ }^{7}$ age groups, ${ }^{8}$ and the use of multiple medications. ${ }^{9}$ Moreover, heterogeneity has been observed in individual safety profiles of benzodiazepine components. ${ }^{10}$

A meta-analysis, integrating four cohort studies and six case-control studies, reported that benzodiazepine users had a $78 \%$ higher potential risk of dementia compared to nonusers. ${ }^{11}$ A subsequent signal detection study using the US Food and Drug Administration Adverse Event Reporting System Database and the Canada Vigilance Adverse Reaction Online Database reported a 1.63-fold and 1.88-fold increased association between benzodiazepine and dementia, respectively. ${ }^{10}$ However, a prospective study found no significant differenc- 
es in the cognitive performance between benzodiazepine users and non-users. ${ }^{12}$

The safety of benzodiazepines has remained uncertain due to the inconsistent results of previous studies and the unknown mechanisms by which benzodiazepine may influence dementia. The case/non-case method, derived from the casecontrol study design used in epidemiology studies, is a pharmacovigilance method validated for signal detection. ${ }^{13} \mathrm{Al}$ though a previous case/non-case study reported an association between benzodiazepine use and memory disorders, ${ }^{14}$ selection bias may have undermined its results. All reports, except memory disorder cases, were selected as non-cases without balancing important demographic features. Our objective was to generate a reliable signal of the association between benzodiazepine use and the potential risk of cognitive impairment or dementia. We aimed to identify susceptible subgroups using a case/non-case method while controlling for the confounding of age and sex.

\section{METHODS}

\section{Data source}

Reported cases related to cognitive impairment or dementia were mined using Korea Institute of Drug Safety \& Risk Management-Korea adverse event reporting system database (KIDS-KD) ${ }^{15}$ KIDS-KD consists of 9 tables: basic information, drug information, ADR information, critical case information, reporter information, causality assessment information, disease history of ADR information, group information, and completeness information. Of 1,089,163 reports accumulated in KIDS-KD between December 1988 and June 2016, 212,127 reports related to benzodiazepine and other medications (opioid analgesics and antidepressants) were identified after excluding cases without age or sex and those younger than 40 years of age (Figure 1). This study was approved by the institutional review board of Sungkyunkwan University (No. 2017-08-008-001), which waived the informed consent, as only deidentified data were used in this study.

\section{Case/non-case approach}

The case/non-case approach measures the disproportionality of combinations between a drug and a particular ADR in a pharmacovigilance database. ${ }^{15-18}$ This approach was chosen to assess the disproportionality of reports for cognitive impairment or dementia associated with benzodiazepines compared to opioid analgesics or antidepressants. Reporting odds ratio (ROR) was calculated as the odds of the exposure to benzodiazepines in comparison with that to other drugs (opioid analgesics, and antidepressants) in cases divided by the odds of the exposure to benzodiazepines in non-cases (Figure 2).
To minimize data duplication, we eliminated reports whose report number, generic name, preferred terms (PTs) and included terms (ITs) according to World Health Organization Adverse Reactions Terminology (WHO-ART) that were duplicated. Each case, defined as a report of cognitive impairment or dementia, was matched to 10 non-cases by age and sex to minimize confounding from these variables. Cognitive impairment or dementia cases were defined using PTs and ITs of the four-level hierarchical system structure of WHOART (adverse reaction record number: “0100," “0199," "1453," and "1877"; Supplementary Table 1 in the online-only Data Supplement). Benzodiazepines were classified as short-, intermediate-, and long-acting according to half-lives; short-acting $<12$ hours; intermediate-acting 12-23 hours; long-acting $\geq 24$. . $^{19,20}$

\section{Causality assessment}

In South Korea, the causality of each adverse event (AE) report from healthcare professionals is evaluated using the modified algorithm based on the Naranjo algorithm developed by WHO-UMC. ${ }^{21}$ Using this algorithm, healthcare professionals including doctors, pharmacists, nurses, and pharmaceutical companies assess causality between drugs and AEs. The evidence level is categorized into six groups: certain, probable/ likely, possible, unlikely, conditional/unclassified, and unassessable. The items of the causality assessment algorithm include the time order between treatment and AEs, outcomes when the drugs were discontinued or re-administered, history of a similar reaction to the same or similar drugs in any previous exposure, concomitant drug use, presence of alternative causes of the reaction, and the presence of already known information on the AEs, based on the product labels or previous case reports.

\section{Potential confounders}

Potential covariates for the association between benzodiazepine use and cognitive impairment or dementia were categorized as age group, sex, year of reporting, polypharmacy, and comorbidities. Polypharmacy was defined as a concomitant prescription of $\geq 5$ drugs. ${ }^{22}$ Comorbidities were coded using the 6th and 7th Korean Standard Classification of Diseases (KCD) and included the following: renal failure, high blood pressure, paranoid schizophrenia, urinary incontinence, constipation, other cholelithiasis, lower back pain, history of major surgery, atrial fibrillation and flutter, bronchopneumonia, normal-pressure hydrocephalus, rheumatoid arthritis, hyperplasia of the prostate, malignant neoplasm of the bronchus or lung, cough, history of malignant neoplasm of digestive organs, complex partial seizures developing into secondarily generalized seizures, migraines, atypical schizophrenia, other 
cerebrovascular diseases, other and unspecified abdominal pain, dysuria, restlessness and agitation, hepatoma, multiple myeloma, unspecified hypertension, spinal stenosis, and headaches.

Additionally, reporting groups were assessed to judge the reliability of the reports. Reporting groups by profession were comprised of doctors, pharmacists, nurses, and consumers, based on information provided about the occupation of the person who made the report of the AEs. Reporting groups by affiliation consisted of hospitals/clinics, pharmacies, manufacturers, and consumers.

\section{Statistical analysis}

Cases of benzodiazepine use, and comparator drug use were compared to determine whether benzodiazepine increases the signal of cognitive impairment or dementia. Logistic regression analysis was conducted to estimate RORs and 95\% confidence intervals (CIs). The models were adjusted for age, sex, year of reporting, polypharmacy, and comorbidities.

Subgroup analysis was performed according to age group, sex, and polypharmacy. Sensitivity analyses were conducted

\begin{tabular}{|l|c|c|}
\hline Exposure & $\begin{array}{c}\text { Cognitive impairment or } \\
\text { dementia cases }\end{array}$ & $\begin{array}{c}\text { Non-dementia } \\
\text { cases }\end{array}$ \\
\hline Benzodiazepines & $\mathrm{A}(\mathrm{N}=56)$ & $\mathrm{B}(\mathrm{N}=289)$ \\
\hline $\begin{array}{l}\text { Others* } \\
* \text { Opioid analgesics and } \\
\text { antidepressants }\end{array}$ & $\mathrm{C}(\mathrm{N}=831)$ & $\mathrm{D}(\mathrm{N}=8,581)$ \\
\hline $\begin{array}{l}\text { Crude ROR }=\frac{\mathrm{A} \times \mathrm{D}}{\mathrm{B} \times \mathrm{C}} \\
\text { 95\% CI }=\mathrm{e}\end{array}$ & \\
\hline
\end{tabular}

Figure 2. RORs and 95\% confidence intervals for cognitive impairment or dementia and the use of benzodiazepines. ROR: reporting odds ratios.

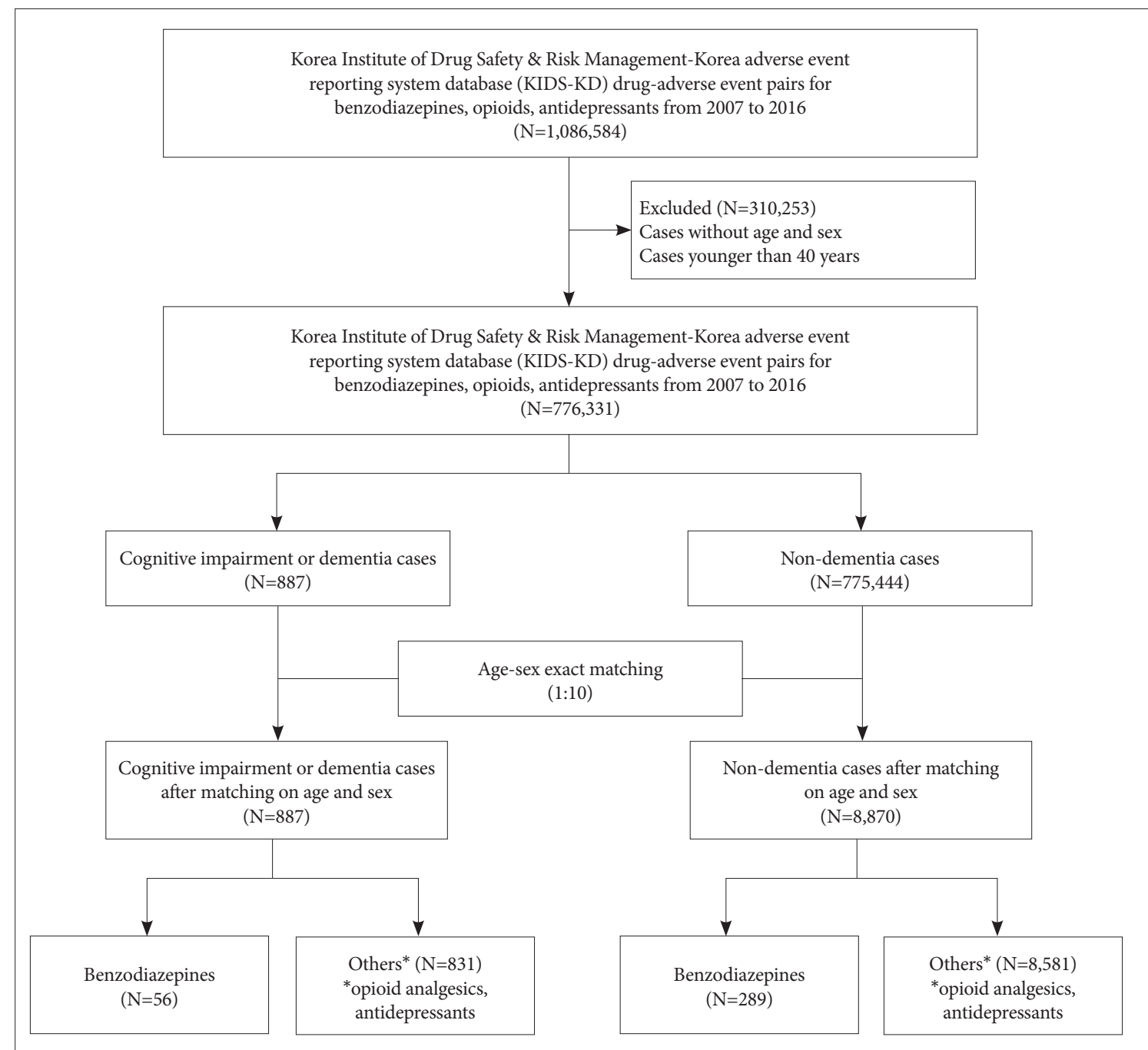

Figure 1. Flow-chart showing the selection of cases and non-cases for the signal evaluation study between the use of benzodiazepine and the risk of cognitive impairment or dementia Korea Institute of Drug Safety \& Risk Management-Korea adverse event reporting system database (KIDS-KD), 2007-2016. 
Table 1. Characteristics of the cases (cognitive impairment or dementia) and non-cases (non-dementia) matched by factors from KIDS-KD, 2007-2016

\begin{tabular}{|c|c|c|c|c|c|c|}
\hline \multirow[b]{2}{*}{ Characteristics } & \multicolumn{3}{|c|}{ Before matching } & \multicolumn{3}{|c|}{ After matching on age and sex (1:10) } \\
\hline & $\begin{array}{c}\text { Cases } \\
\mathrm{N}=887(\%)\end{array}$ & $\begin{array}{c}\text { Non-cases } \\
\mathrm{N}=775,444(\%)\end{array}$ & $\mathrm{p}$-value & $\begin{array}{c}\text { Cases } \\
\mathrm{N}=887(\%)\end{array}$ & $\begin{array}{c}\text { Non-cases } \\
\mathrm{N}=8,870(\%)\end{array}$ & p-value \\
\hline Age group & & & $<0.001$ & & & 1.00 \\
\hline $40-44$ & $54(6.1)$ & $60,596(7.8)$ & & $54(6.1)$ & $540(6.1)$ & \\
\hline $45-49$ & $48(5.4)$ & $83,930(10.8)$ & & $48(5.4)$ & $480(5.4)$ & \\
\hline $50-54$ & $26(2.9)$ & $120,610(15.6)$ & & $26(2.9)$ & $260(2.9)$ & \\
\hline $55-59$ & $133(15.0)$ & $104,767(13.5)$ & & $133(15.0)$ & $1,330(15.0)$ & \\
\hline $60-64$ & $171(19.3)$ & $96,275(12.4)$ & & $171(19.3)$ & $1,710(19.3)$ & \\
\hline $65-69$ & $103(11.6)$ & $105,472(13.6)$ & & $103(11.6)$ & $1,030(11.6)$ & \\
\hline $70-74$ & $87(9.8)$ & $98,039(12.6)$ & & $87(9.8)$ & $870(8.8)$ & \\
\hline $75-79$ & $174(19.6)$ & $63,566(8.2)$ & & $174(19.6)$ & $1,740(19.6)$ & \\
\hline $80-84$ & $28(3.2)$ & $31,716(4.1)$ & & $28(3.2)$ & $280(3.2)$ & \\
\hline$\geq 85$ & $63(7.1)$ & $10,473(1.4)$ & & $63(7.1)$ & $630(7.1)$ & \\
\hline Sex & & & $<0.078$ & & & 1.00 \\
\hline Male & $417(47.0)$ & $341,503(44.0)$ & & $417(47.0)$ & $4,170(47.0)$ & \\
\hline Female & $470(53.0)$ & $433,941(56.0)$ & & $470(53.0)$ & $4,700(53.0)$ & \\
\hline Reporting group by profession & & & $<0.001$ & & & $<0.001$ \\
\hline Doctor & $465(52.4)$ & $389,733(50.3)$ & & $465(52.4)$ & $4,814(54.3)$ & \\
\hline Pharmacist & $81(9.1)$ & $57,138(7.4)$ & & $81(9.1)$ & $646(7.3)$ & \\
\hline Nurse & $78(8.8)$ & $155,761(20.1)$ & & $78(8.8)$ & $1,781(20.1)$ & \\
\hline Consumer & $12(1.4)$ & $5,211(0.7)$ & & $12(1.4)$ & $63(0.7)$ & \\
\hline Other healthcare professional & $49(5.5)$ & $45,446(5.9)$ & & $49(5.5)$ & $555(6.3)$ & \\
\hline Reporting group by affiliation & & & $<0.001$ & & & $<0.001$ \\
\hline Hospitals/clinics & $254(28.6)$ & $313,700(40.5)$ & & $254(28.6)$ & $3,628(40.9)$ & \\
\hline Pharmacies & $10(1.1)$ & $13,389(1.7)$ & & $10(1.1)$ & $134(1.5)$ & \\
\hline Manufacturers & $621(70.0)$ & $447,112(57.7)$ & & $621(70.0)$ & $5,096(57.5)$ & \\
\hline Consumers & $2(0.2)$ & $850(0.1)$ & & $2(0.2)$ & $7(0.1)$ & \\
\hline Others & $0(0.0)$ & $393(0.1)$ & & $0(0.0)$ & $5(0.1)$ & \\
\hline Polypharmacy $(\geq 5)$ & & & $<0.078$ & & & $<0.190$ \\
\hline Yes & $665(75.0)$ & $560,564(72.3)$ & & $665(75.0)$ & $6465(72.9)$ & \\
\hline No & $222(25.0)$ & $214,880(27.7)$ & & $222(25.0)$ & $2405(27.1)$ & \\
\hline \multicolumn{7}{|l|}{ Comorbidities } \\
\hline Renal failure & $69(7.8)$ & $1,690(0.2)$ & $<0.001$ & $69(7.8)$ & $23(0.3)$ & $<0.001$ \\
\hline Hypertension & $53(6.0)$ & $8,028(1.0)$ & $<0.001$ & $53(6.0)$ & $90(1.0)$ & $<0.001$ \\
\hline Paranoid Schizophrenia & $38(4.3)$ & $528(0.1)$ & $<0.001$ & $38(4.3)$ & $6(0.1)$ & $<0.001$ \\
\hline Urinary incontinence & $35(3.9)$ & $561(0.1)$ & $<0.001$ & $35(3.9)$ & $7(0.1)$ & $<0.001$ \\
\hline Constipation & $29(3.3)$ & $2,044(0.3)$ & $<0.001$ & $29(3.3)$ & $22(0.2)$ & $<0.001$ \\
\hline Other cholelithiasis & $29(3.3)$ & $1,339(0.2)$ & $<0.001$ & $29(3.3)$ & $33(0.4)$ & $<0.001$ \\
\hline Low back pain & $21(2.4)$ & $2,022(0.3)$ & $<0.001$ & $21(2.4)$ & $221(2.5)$ & $<0.821$ \\
\hline History of major surgery & $21(2.4)$ & $10,572(1.4)$ & $<0.010$ & $21(2.4)$ & $306(3.4)$ & $<0.088$ \\
\hline $\mathrm{AFF}$ & $14(1.6)$ & $109(0.0)$ & $<0.001$ & $14(1.6)$ & $1(0.0)$ & $<0.001$ \\
\hline Bronchopneumonia & $14(1.6)$ & $57(0.0)$ & $<0.001$ & $14(1.6)$ & $3(0.0)$ & $<0.001$ \\
\hline $\mathrm{NPH}$ & $13(1.5)$ & $35(0.0)$ & $<0.001$ & $13(1.5)$ & $1(0.0)$ & $<0.001$ \\
\hline $\mathrm{RA}$ & $12(1.4)$ & $1,059(0.1)$ & $<0.001$ & $12(1.4)$ & $17(0.2)$ & $<0.001$ \\
\hline Hyperplasia of prostate & $12(1.4)$ & $7,214(0.9)$ & $<0.190$ & $12(1.4)$ & $74(0.8)$ & $<0.115$ \\
\hline Lung cancer & $11(1.2)$ & $4,891(0.6)$ & $<0.022$ & $11(1.2)$ & $54(0.6)$ & $<0.028$ \\
\hline Cough & $10(1.1)$ & $1,059(0.1)$ & $<0.001$ & $10(1.1)$ & $11(0.1)$ & $<0.001$ \\
\hline
\end{tabular}


Table 1. Characteristics of the cases (cognitive impairment or dementia) and non-cases (non-dementia) matched by factors from KIDS-KD, 2007-2016 (continued)

\begin{tabular}{|c|c|c|c|c|c|c|}
\hline \multirow[b]{2}{*}{ Characteristics } & \multicolumn{3}{|c|}{ Before matching } & \multicolumn{3}{|c|}{ After matching on age and sex $(1: 10)$} \\
\hline & $\begin{array}{c}\text { Cases } \\
\mathrm{N}=887(\%)\end{array}$ & $\begin{array}{c}\text { Non-cases } \\
\mathrm{N}=775,444(\%)\end{array}$ & $\mathrm{p}$-value & $\begin{array}{c}\text { Cases } \\
\mathrm{N}=887(\%)\end{array}$ & $\begin{array}{c}\text { Non-cases } \\
\mathrm{N}=8,870(\%)\end{array}$ & p-value \\
\hline Year of reporting & & & $<0.002$ & & & $<0.093$ \\
\hline 2007 & $28(3.2)$ & $50,401(6.5)$ & & $28(3.2)$ & $206(2.3)$ & \\
\hline 2008 & $16(1.8)$ & $8,821(1.1)$ & & $16(1.8)$ & $111(1.3)$ & \\
\hline 2009 & $35(3.9)$ & $12,410(1.6)$ & & $35(3.9)$ & $150(1.7)$ & \\
\hline 2010 & $107(12.1)$ & $35,558(4.6)$ & & $107(12.1)$ & $378(4.3)$ & \\
\hline 2011 & $35(3.9)$ & $37,978(4.9)$ & & $35(3.9)$ & $452(5.1)$ & \\
\hline 2012 & $17(1.9)$ & $53,666(6.9)$ & & $17(1.9)$ & $622(7.0)$ & \\
\hline 2013 & $129(14.5)$ & $180,911(23.3)$ & & $129(14.5)$ & $1,891(21.3)$ & \\
\hline 2014 & $23(2.6)$ & $46,587(6.0)$ & & $23(2.6)$ & $537(6.1)$ & \\
\hline 2015 & $155(17.5)$ & $171,127(22.1)$ & & $155(17.5)$ & $2,239(25.2)$ & \\
\hline 2016 & $342(38.6)$ & $177,985(23.0)$ & & $342(38.6)$ & $2,284(25.7)$ & \\
\hline
\end{tabular}

KIDS-KD: Korea Institute of Drug Safety \& Risk Management-Korea adverse event reporting system database, AFF: atrial fibrillation and flutter, $\mathrm{NPH}$ : normal pressure hydrocephalus, RA: rheumatoid arthritis

Table 2. Disproportionality analysis of cognitive impairment or dementia associated with the use of benzodiazepines in all adverse events and applied to reports with certain, probable, and possible causality assessments from KIDS-KD, 2007-2016

\begin{tabular}{|c|c|c|c|c|c|c|c|c|c|c|c|c|}
\hline & \multicolumn{6}{|c|}{ Before matching } & \multicolumn{6}{|c|}{ After matching on age and sex $(1: 10)$} \\
\hline & \multirow[b]{2}{*}{ ROR } & \multicolumn{2}{|c|}{$95 \% \mathrm{CI}$} & \multirow{2}{*}{$\begin{array}{c}\text { Adjusted } \\
\text { ROR}^{*}\end{array}$} & \multicolumn{2}{|c|}{$95 \% \mathrm{CI}$} & \multirow[b]{2}{*}{ ROR } & \multicolumn{2}{|c|}{$95 \% \mathrm{CI}$} & \multirow{2}{*}{$\begin{array}{c}\text { Adjusted } \\
\text { ROR* }\end{array}$} & \multicolumn{2}{|c|}{$95 \% \mathrm{CI}$} \\
\hline & & $\begin{array}{l}\text { Lower } \\
\text { limit }\end{array}$ & $\begin{array}{l}\text { Upper } \\
\text { limit }\end{array}$ & & $\begin{array}{c}\text { Lower } \\
\text { limit }\end{array}$ & $\begin{array}{l}\text { Upper } \\
\text { limit }\end{array}$ & & $\begin{array}{c}\text { Lower } \\
\text { limit }\end{array}$ & $\begin{array}{l}\text { Upper } \\
\text { limit }\end{array}$ & & $\begin{array}{c}\text { Lower } \\
\text { limit }\end{array}$ & $\begin{array}{c}\text { Upper } \\
\text { limit }\end{array}$ \\
\hline \multicolumn{13}{|c|}{ Including all causality assessments } \\
\hline Benzodiazepine & 1.98 & 1.51 & 2.59 & 2.04 & 1.55 & 2.68 & 2.00 & 1.49 & 2.69 & 2.23 & 1.61 & 3.09 \\
\hline \multicolumn{13}{|c|}{ Restricted to adverse events with causality recorded as certain, probable, and/or possible } \\
\hline Benzodiazepine & 2.85 & 1.87 & 4.35 & 2.70 & 1.76 & 4.12 & 2.58 & 1.62 & 4.12 & 2.69 & 1.66 & 4.38 \\
\hline
\end{tabular}

*adjusted according to age group, sex, year of reporting, polypharmacy, and comorbidity. KIDS-KD: Korea Institute of Drug Safety \& Risk Management-Korea adverse event reporting system database, ROR: reporting odds ratio, 95\% CI: 95\% confidence interval

to assess bias in the causality of reports. Reports with causality assessments of "certain," "probable," and "possible" were analyzed and the total number of cases was readjusted to 249,640 , with 243 cognitive impairment or dementia cases and 249,397 non-cases. After 1:10 matching, 243 cases, and 2,430 non-cases were included. All statistical procedures were performed using the SAS 9.4 software (SAS Institute Inc., Cary, NC, USA). A two-tailed value of $\mathrm{p}<0.05$ indicated statistical significance.

\section{RESULTS}

From January 2007 to December 2016, there were 1,086,584 AE-pairs pairs involving benzodiazepines that were identified. A total of 776,331 AE-pairs were included after excluding cases without age or sex $(n=162,677)$ and those younger than 40 years of age $(n=147,576)$. There were 887 cognitive impairment or dementia AE-pairs defined as cases, and 775,444 other AE-pairs were defined as non-cases. After 1:10 match- ing according to age and sex, 887 cases and 8,870 non-cases were included.

Table 1 shows the characteristics of cases and non-cases; $75.9 \%$ and $87.9 \%$ of cognitive impairment or dementia cases and matched non-cases, respectively, were reported by healthcare professionals. Among the cases, disproportionality was found after any use of benzodiazepine as compared with nonuse. There was a difference in the magnitude of association before matching (adjusted ROR, aROR $=2.04$; 95\% CI $=1.55^{-}$ $2.68)$ and after matching $(\mathrm{aROR}=2.23$; 95\% $\mathrm{CI}=1.61-3.09)$. Sensitivity analysis was implemented to limit AEs to those with certain, probable, or possible causality assessments; this restriction increased the disproportionality of benzodiazepine use $(\mathrm{aROR}=2.69 ; 95 \% \mathrm{CI}=1.66-4.38)$ compared to that observed across all AEs (Table 2). Individual benzodiazepine drugs had heterogeneous levels of safety profiles for cognitive impairment or dementia (Table 3).

In the subgroup analysis (Table 4), there was no significant 
difference in RORs among different age groups. Higher ROR estimates were observed in female $(\mathrm{aROR}=2.33,95 \% \mathrm{CI}=$ $1.48-3.67)$ than in male $(\mathrm{aROR}=1.89,95 \% \mathrm{CI}=1.11-3.20)$, and in those with polypharmacy $(\mathrm{aROR}=2.20,95 \% \mathrm{CI}=1.35-$ 3.57 ) than in those without polypharmacy ( $\mathrm{aROR}=2.06,95 \%$ $\mathrm{CI}=1.30-3.27)$. However, the subgroup difference was not statistically significant in the interaction test.

\section{DISCUSSION}

This case/non-case study using 10 years of spontaneous nationwide reports showed that benzodiazepine use was associated with a potential risk of cognitive impairment or dementia. Among the subgroups, higher RORs were observed in female and those who had polypharmacy. The possible asso-

Table 3. Disproportionality analysis of cognitive impairment or dementia associated with the use of individual benzodiazepines from KIDSKD, 2007-2016*

\begin{tabular}{|c|c|c|c|c|c|c|c|c|c|c|c|c|}
\hline & \multicolumn{6}{|c|}{ Before matching } & \multicolumn{6}{|c|}{ After matching on age and sex (1:10) } \\
\hline & \multirow[b]{2}{*}{ ROR } & \multicolumn{2}{|c|}{$95 \% \mathrm{CI}$} & \multirow{2}{*}{$\begin{array}{c}\text { Adjusted } \\
\mathrm{ROR}^{\dagger}\end{array}$} & \multicolumn{2}{|c|}{$95 \%$ CI } & \multirow[b]{2}{*}{ ROR } & \multicolumn{2}{|c|}{$95 \% \mathrm{CI}$} & \multirow{2}{*}{$\begin{array}{c}\text { Adjusted } \\
\mathrm{ROR}^{\dagger}\end{array}$} & \multicolumn{2}{|c|}{$95 \%$ CI } \\
\hline & & $\begin{array}{l}\text { Lower } \\
\text { limit }\end{array}$ & $\begin{array}{l}\text { Upper } \\
\text { limit }\end{array}$ & & $\begin{array}{l}\text { Lower } \\
\text { limit }\end{array}$ & $\begin{array}{l}\text { Upper } \\
\text { limit }\end{array}$ & & $\begin{array}{l}\text { Lower } \\
\text { limit }\end{array}$ & $\begin{array}{l}\text { Upper } \\
\text { limit }\end{array}$ & & $\begin{array}{l}\text { Lower } \\
\text { limit }\end{array}$ & $\begin{array}{c}\text { Upper } \\
\text { limit }\end{array}$ \\
\hline \multicolumn{13}{|c|}{ Short-acting benzodiazepines } \\
\hline Etizolam & 1.36 & 0.42 & 4.36 & 1.55 & 0.48 & 5.03 & 1.20 & 0.33 & 4.36 & 1.26 & 0.28 & 5.64 \\
\hline Midazolam & 0.49 & 0.21 & 1.15 & 0.44 & 0.18 & 1.05 & 0.46 & 0.19 & 1.12 & 0.36 & 0.13 & 1.00 \\
\hline Tofisopam & 1.59 & 0.22 & 11.54 & 1.53 & 0.20 & 11.44 & 0.73 & 0.09 & 6.07 & 0.76 & 0.08 & 7.33 \\
\hline Triazolam & 0.77 & 0.19 & 3.15 & 0.73 & 0.17 & 3.03 & 0.86 & 0.19 & 3.93 & 0.77 & 0.15 & 4.04 \\
\hline \multicolumn{13}{|c|}{ Intermediate-acting benzodiazepines } \\
\hline Alprazolam & 1.13 & 0.60 & 2.14 & 1.23 & 0.64 & 2.36 & 1.24 & 0.61 & 2.52 & 1.72 & 0.75 & 3.97 \\
\hline Bromazepam & 3.31 & 0.46 & 24.05 & 3.16 & 0.42 & 23.91 & 2.61 & 0.23 & 29.28 & 1.56 & 0.12 & 20.17 \\
\hline Flunitrazepam & 8.75 & 2.11 & 36.31 & 7.32 & 1.64 & 32.58 & 2.64 & 0.47 & 14.77 & 1.38 & 0.15 & 12.53 \\
\hline Lorazepam & 1.53 & 0.88 & 2.67 & 1.64 & 0.93 & 2.90 & 1.92 & 1.03 & 3.57 & 1.67 & 0.81 & 3.43 \\
\hline \multicolumn{13}{|c|}{ Long-acting benzodiazepines } \\
\hline Clonazepam & 0.81 & 0.32 & 2.03 & 0.71 & 0.28 & 1.79 & 0.61 & 0.23 & 1.62 & 0.72 & 0.24 & 2.16 \\
\hline Diazepam & 0.79 & 0.32 & 1.98 & 0.80 & 0.32 & 2.02 & 0.88 & 0.33 & 2.38 & 1.05 & 0.32 & 3.43 \\
\hline
\end{tabular}

*analysis based on logistic on analysis, reference group: other benzodiazepines. This table displays only benzodiazepines referenced in over 100 reports, ${ }^{\dagger}$ adjusted according to age group, sex, year of reporting, and polypharmacy. KIDS-KD: Korea Institute of Drug Safety \& Risk Management-Korea adverse event reporting system database, ROR: reporting odds ratio, 95\% CI: 95\% confidence interval

Table 4. Subgroup analysis associated with factors for cognitive impairment or dementia: age (resetting for higher causality on cognitive impairment or dementia), sex, and polypharmacy from KIDS-KD, 2007-2016

\begin{tabular}{|c|c|c|c|c|c|c|c|c|c|c|c|c|}
\hline & \multicolumn{6}{|c|}{ Before matching } & \multicolumn{6}{|c|}{ After matching on age and sex $(1: 10)$} \\
\hline & \multirow[b]{2}{*}{ ROR } & \multicolumn{2}{|c|}{$95 \%$ CI } & \multirow{2}{*}{$\begin{array}{c}\text { Adjusted } \\
\text { ROR }\end{array}$} & \multicolumn{2}{|c|}{$95 \% \mathrm{CI}$} & \multirow[b]{2}{*}{ ROR } & \multicolumn{2}{|c|}{$95 \% \mathrm{CI}$} & \multirow{2}{*}{$\begin{array}{c}\text { Adjusted } \\
\text { ROR }\end{array}$} & \multicolumn{2}{|c|}{$95 \% \mathrm{CI}$} \\
\hline & & $\begin{array}{c}\text { Lower } \\
\text { limit }\end{array}$ & $\begin{array}{c}\text { Upper } \\
\text { limit }\end{array}$ & & $\begin{array}{c}\text { Lower } \\
\text { limit }\end{array}$ & $\begin{array}{c}\text { Upper } \\
\text { limit }\end{array}$ & & $\begin{array}{c}\text { Lower } \\
\text { limit }\end{array}$ & $\begin{array}{l}\text { Upper } \\
\text { limit }\end{array}$ & & $\begin{array}{c}\text { Lower } \\
\text { limit }\end{array}$ & $\begin{array}{c}\text { Upper } \\
\text { limit }\end{array}$ \\
\hline \multicolumn{13}{|c|}{ Age group* } \\
\hline$\leq 64$ & 2.13 & 1.47 & 3.09 & 1.80 & 1.21 & 2.68 & 1.92 & 1.28 & 2.87 & 2.37 & 1.46 & 3.84 \\
\hline $65-74$ & 1.77 & 0.94 & 3.35 & 1.69 & 0.88 & 3.25 & 2.19 & 1.09 & 4.41 & 2.22 & 1.04 & 4.75 \\
\hline$\geq 75$ & 1.80 & 1.08 & 2.98 & 2.24 & 1.32 & 3.82 & 2.06 & 1.19 & 3.59 & 2.33 & 1.22 & 4.44 \\
\hline \multicolumn{13}{|l|}{$\operatorname{Sex}^{\dagger}$} \\
\hline Male & 1.88 & 1.24 & 2.84 & 1.51 & 0.96 & 2.38 & 1.78 & 1.14 & 2.79 & 1.89 & 1.11 & 3.20 \\
\hline Female & 2.07 & 1.44 & 2.96 & 2.15 & 1.48 & 3.11 & 2.20 & 1.49 & 3.26 & 2.33 & 1.48 & 3.67 \\
\hline \multicolumn{13}{|c|}{ Polypharmacy $\ddagger$} \\
\hline Yes & 1.67 & 1.17 & 2.40 & 1.57 & 1.08 & 2.29 & 1.95 & 1.32 & 2.89 & 2.20 & 1.35 & 3.57 \\
\hline No & 2.69 & 1.77 & 4.08 & 2.47 & 1.62 & 3.75 & 2.20 & 1.40 & 3.46 & 2.06 & 1.30 & 3.27 \\
\hline
\end{tabular}

*adjusted for sex, polypharmacy, and comorbidity, ${ }^{\dagger}$ adjusted for age group, polypharmacy, and comorbidity, ${ }^{\ddagger}$ adjusted for age group, sex, and comorbidity. KIDS-KD: Korea Institute of Drug Safety \& Risk Management-Korea adverse event reporting system database, ROR: reporting odds ratio, 95\% CI: 95\% confidence interval 
ciation was supported by the disproportionality analysis, in which the magnitude of ROR increased when the analysis was restricted to reports suspected to have associations in the causality assessment by healthcare professionals. Benzodiazepine use was associated with a 2.69-fold increased report of cognitive impairment or dementia when assessed using reports with certain, probable, and/or possible in causality assessments. Heterogeneity was observed in the individual safety profile of benzodiazepine components.

Despite being statistically insignificant, higher ROR estimates for cognitive decline/dementia in females and those with polypharmacy suggest that these subgroups are more susceptible to benzodiazepine-associated dementia risk. Similar findings that support our results are in a previously reported systematic review which showed a higher association between benzodiazepine use and dementia among females (Odds ratio $=1.16,95 \% \mathrm{CI}=1.00-1.36)$ than in males $(1.00,0.96-1.04) .{ }^{11}$ Patients with polypharmacy have a particularly increased risk of benzodiazepine-induced cognitive impairment because benzodiazepines may trigger drug-drug interactions when concomitantly used with other drugs metabolized by CYP3A. ${ }^{23}$

There was no difference in ROR estimates among different age groups. This can be due to the depletion of susceptible effects in older patients, ${ }^{24}$ in which these patients already at a high risk of dementia from other events associated with dementia such as neurological and psychiatric disorders, leading to a non-differential ROR compared to younger groups. We observed a heterogeneity in the risk of individual benzodiazepine drugs, in contrast to the findings from the US FAERS database where the ROR values increased with the duration of benzodiazepine action. This inconsistency may be due to the discrepancies in baseline characteristics among the users of different benzodiazepine components in our database or the difference in clinical indications.

Although the mechanism of the influence of benzodiazepine on dementia is unknown, the following hypotheses support our results. First, benzodiazepine has neuro-sedative effects and causes cognitive deficits, which may lead to a decrease in cognitive reserve when used chronically. ${ }^{25,26}$ Second, chronic use of benzodiazepines could exacerbate dementia by downregulating gamma-aminobutyric acid (GABA) receptors. ${ }^{27,28}$ Finally, benzodiazepine use may increase the potential risk of cognitive impairment in early dementia because of the GABA-secreting activity expressed in amyloid plaques. ${ }^{29}$

Our observations are in line with previous pharmacoepidemiologic studies reporting increasing potential risks among benzodiazepine users. ${ }^{3-5,30,31}$ The results are also consistent with those of a signal detection study conducted in Japan on the association between benzodiazepine use and the potential risk of dementia. ${ }^{10}$ A previous case/non-case study showed a greater association than did our study, reporting a more than 6 -fold increased reports of memory disorder among benzodiazepine users. ${ }^{14}$ Contrary to this, some cohort studies observed no association between benzodiazepine use and dementia. ${ }^{32,33}$

Our results are subject to reverse causation owing to the limitations of the AE reporting system database, which lacks information on the presence of biologically plausible lag time between benzodiazepine initiation and the occurrence of dementia to consider disease latency. Provided that dementia has a latent period of several years and insidious onset, it is possible that benzodiazepines were prescribed for prodromal symptoms of dementia. However, given that over $75 \%$ of cognitive impairment or dementia reports were from healthcare professionals in our database and the magnitude of ROR increased when the analysis was restricted to reports suspected to have association in the causality assessment, our signal indicates a possible association between benzodiazepine and the risk of cognitive impairment or dementia.

Our study has several strengths. We used a nationwide spontaneous reporting database, which accumulated 1,086,564 AE reports over 10 years. Second, the case/non-case design has an advantage in the feasibility of using demographic and clinical variables in the analyses, which has been a limitation of traditional signal detection methods, such as proportional reporting ratios, RORs, and information components. Since increasing age and female sex are known risk factors for dementia, cases and non-cases were matched on these factors to exclude potential confounding. Matching is the most effective technique to increase comparability between cases and non-cases because it ensures equal distributions of matching variables. We also performed multivariate analyses to control other confounders. Given that benzodiazepines are one of the most commonly used psychotropic drugs in elderly patients, our findings have important public health implications for this population.

However, our study should be interpreted with caution. First, the limitations of spontaneous reporting may limit causal interpretation of our findings, as such reports are subject to the subjective judgments of the reporter. Second, our results are subject to protopathic bias and may have been influence by uncontrolled confounders as the database lacks clinical or demographic information. Important epidemiologic factors associated with dementia, such as depression, low education level, and smoking were not controlled in the analyses. ${ }^{34}$ Our analyses also did not reflect the influence of alcohol consumption, which reportedly increases the potential risk of cognitive impairment 3.6-fold, ${ }^{1}$ or body mass index (BMI). Obese patients $(\mathrm{BMI}>30)$ had a $74 \%$ increased risk of dementia compared to those with average weight. ${ }^{35}$ Third, the imbalances of baseline characteristics between cases and non-cases 
may lead to the ROR overestimations for benzodiazepine use and dementia. For example, the higher prevalence of paranoid schizophrenia among cases in which benzodiazepine may be used for the treatment may lead to a higher benzodiazepine prevalence and consequent ROR overestimation. ${ }^{36}$ Although we adjusted the comorbidities in the outcome model, the imbalance of confounders between cases and non-cases may have resulted in ROR overestimations. Cognitive decline and dementia can be clinically different conditions. The difference between these two conditions lies in whether the patient has impaired activities of daily living. ${ }^{37}$ Due to the inherent limitations of a retrospective study, it remains unclear how the clinical judgement differentiated cognitive decline from dementia. Nonetheless, we used both cognitive decline and dementia to define a case since these two conditions share common symptoms. Further, cognitive impairment can be a prodromal stage of dementia. ${ }^{38}$

In conclusion, our case/non-case study detected an increased association for cognitive impairment or dementia in female and in those with polypharmacy associated with benzodiazepine use from a 10-year, cumulated AE database. We found inconsistencies in dementia safety profiles across benzodiazepine components, which warrant further investigations. South Korea has a rapidly aging population, which is a non-modifiable potential risk factor for dementia. ${ }^{39}$ In this regard, the high prevalence of benzodiazepine use and its potential association with dementia are an important public health concern. We recommend further pharmacoepidemiologic studies to confirm our findings.

\section{Supplementary Materials}

The online-only Data Supplement is available with this article at https://doi.org/10.30773/pi.2019.0275.

\section{Acknowledgments}

This work was supported by the National Research Foundation of Korea (NRF) grant funded by the Korea government (Ministry of Science and Information \& Communication Technology, MSIT) (No. NRF-2020R1C1C 1003527). This work was supported by the Korea Foundation for the Advancement of Science \& Creativity (KOFAC), and funded by the Korean Government (MOE). We sincerely thank the Korea Institute of Drug Safety \& Risk Management (Ministry of Food and Drug Safety) for their cooperation in providing access to the Korean Institute of Drug Safety \& Risk Management-Korea adverse event reporting system database (KIDS-KD). All authors declare no conflicts of interest.

\section{Conflicts of Interest}

The authors have no potential conflicts of interest to disclose.

\section{Author Contributions}

Conceptualization: Yeon-Hee Baek, Hye-Jun Kim, Ju-Young Shin. Data curation: Hye-Jun Kim. Formal analysis: Hye-Jun Kim, Hyesung Lee, In-Sun Oh. Funding acquisition: Ju-Young Shin. Investigation: Yeon-Hee Baek, Hey-Jun Kim. Methodology: Yeon-Hee Baek, Hye-Jun Kim, JY Shin. Project administration: Yeon-Hee Baek. Resources: Ju-Young Shin. Software:
Hye-Jun Kim, Hyesung Lee, In-Sun Oh. Supervision: Ju-Young Shin. Validation: Hye-Jun Kim. Visualization: Yeon-Hee Baek, Hye-Jun Kim. Writing_original draft: Yeon-Hee Baek, Hye-Jun Kim. Writing_review \& editing: Yeon-Hee Baek, Ji-Hwan Bae, Woo Jung Kim, Ju-Young Shin.

\section{ORCID iDs}

Yeon-Hee Baek

Hye-Jun Kim

Ji-Hwan Bae

Hyesung Lee

In-Sun Oh

Woo Jung Kim

Ju-Young Shin

\section{REFERENCES}

1. Anttila T, Helkala EL, Viitanen M, Kareholt I, Fratiglioni L, Winblad B, et al. Alcohol drinking in middle age and subsequent risk of mild cognitive impairment and dementia in old age: a prospective population based study. BMJ Brit Med J 2004;329:539.

2. Donoghue J, Lader M. Usage of benzodiazepines: a review. Int J Psychiatry Clin 2010;14:78-87.

3. Gray SL, Dublin S, Yu O, Walker R, Anderson M, Hubbard RA, et al. Benzodiazepine use and risk of incident dementia or cognitive decline: prospective population based study. BMJ Brit Med J 2016;352:190.

4. Billioti de Gage S, Begaud B, Bazin F, Verdoux H, Dartigues JF, Peres K, et al. Benzodiazepine use and risk of dementia: prospective population based study. BMJ Brit Med J 2012;345:e6231.

5. Billioti de Gage S, Moride Y, Ducruet T, Kurth T, Verdoux H, Tournier $\mathrm{M}$, et al. Benzodiazepine use and risk of Alzheimer's disease: case-control study. BMJ 2014;349:g5205.

6. Puustinen J, Nurminen J, Vahlberg T, Lyles A, Isoaho R, Raiha I, et al. CNS medications as predictors of precipitous cognitive decline in the cognitively disabled aged: a longitudinal population-based study. Dement Geriatr Cogn Dis Extra 2012;2:57-68.

7. Azad NA, Al Bugami M, Loy-English I. Gender differences in dementia risk factors. Gender Med 2007;4:120-129.

8. van der Flier WM, Scheltens P. Epidemiology and risk factors of dementia. J Neurol Neurosur Psychiatry 2005;76 (Suppl 5):v2-v7.

9. Lai SW, Lin CH, Liao KF, Su LT, Sung FC, Lin CC. Association between polypharmacy and dementia in older people: a population-based casecontrol study in Taiwan. Geriatr Gerontol Int 2012;12:491-498.

10. Takada M, Fujimoto M, Hosomi K. Association between benzodiazepine use and dementia: data mining of different medical databases. Int $\mathrm{J}$ Med Sci 2016;13:825-834.

11. Islam MM, Iqbal U, Walther B, Atique S, Dubey NK, Nguyen PA, et al. Benzodiazepine use and risk of dementia in the elderly population: a systematic review and meta-analysis. Neuroepidemiology 2016;47:181191.

12. Grande G, Tramacere I, Vetrano DL, Pomati S, Mariani C, Filippini G. Use of benzodiazepines and cognitive performance in primary care patients with first cognitive complaints. Int Psychogeriatr 2018;30:597-601.

13. Montastruc JL, Sommet A, Bagheri H, Lapeyre-Mestre M. Benefits and strengths of the disproportionality analysis for identification of adverse drug reactions in a pharmacovigilance database. Br J Clin Pharmacol 2011;72:905-908.

14. Chavant F, Favreliere S, Lafay-Chebassier C, Plazanet C, Perault-Pochat MC. Memory disorders associated with consumption of drugs: updating through a case/noncase study in the French PharmacoVigilance Database. Br J Clin Pharmacol 2011;72:898-904.

15. Shin JY, Jung SY, Ahn SH, Lee SH, Kim SJ, Seong JM, et al. New initiatives for pharmacovigilance in South Korea: introducing the Korea Institute of Drug Safety and Risk Management (KIDS). Pharmacoepidemiol Drug Saf 2014;23:1115-1122.

16. Egberts AC, Meyboom RH, De Koning FH, Bakker A, Leufkens HG. 
Non-puerperal lactation associated with antidepressant drug use. Br J Clin Pharmacol 1997;44:277-281.

17. Egberts AC, Meyboom RH, van Puijenbroek EP. Use of measures of disproportionality in pharmacovigilance: three Dutch examples. Drug Saf 2002;25:453-458.

18. Stricker BH, Tijssen JG. Serum sickness-like reactions to cefaclor. J Clin Epidemiol 1992;45:1177-1184.

19. Estivill E, Bove A, Garcia-Borreguero D, Gibert J, Paniagua J, Pin G, et al. Consensus on drug treatment, definition and diagnosis for insomnia. Clin Drug Investig 2003;23:351-385.

20. Nomura K, Nakao M, Sato M, Yano E. Regular prescriptions for benzodiazepines: a cross-sectional study of outpatients at a university hospital. Intern Med 2006;45:1279-1283.

21. Murayama H, Sakuma M, Takahashi Y, Morimoto T. Improving the assessment of adverse drug reactions using the Naranjo Algorithm in daily practice: the Japan Adverse Drug Events Study. Pharmacol Res Perspect 2018;6.

22. Haider SI, Johnell K, Thorslund M, Fastbom J. Trends in polypharmacy and potential drug-drug interactions across educational groups in elderly patients in Sweden for the period 1992-2002. Int J Clin Pharmacol Ther 2007;45:643-653.

23. Altamura AC, Moliterno D, Paletta S, Maffini M, Mauri MC, Bareggi S. Understanding the pharmacokinetics of anxiolytic drugs. Expert Opin Drug Metab Toxicol 2013;9:423-440.

24. Moride Y, Abenhaim L. Evidence of the depletion of susceptibles effect in non-experimental pharmacoepidemiologic research. J Clin Epidemiol 1994;47:731-737.

25. Stranks EK, Crowe SF. The acute cognitive effects of zopiclone, zolpidem, zaleplon, and eszopiclone: a systematic review and meta-analysis. J Clin Exp Neuropsychol 2014;36:691-700.

26. Tannenbaum C, Paquette A, Hilmer S, Holroyd-Leduc J, Carnahan R. A systematic review of amnestic and non-amnestic mild cognitive impairment induced by anticholinergic, antihistamine, GABAergic and opioid drugs. Drugs Aging 2012;29:639-658.

27. Hutchinson MA, Smith PF, Darlington CL. The behavioural and neuronal effects of the chronic administration of benzodiazepine anxiolytic and hypnotic drugs. Prog Neurobiol 1996;49:73-97.
28. Limon A, Reyes-Ruiz JM, Miledi R. Loss of functional GABA(A) receptors in the Alzheimer diseased brain. Proc Natl Acad Sci USA 2012;109: 10071-10076.

29. Jo S, Yarishkin O, Hwang YJ, Chun YE, Park M, Woo DH, et al. GABA from reactive astrocytes impairs memory in mouse models of Alzheimer's disease. Nat Med 2014;20:886-896.

30. Lagnaoui R, Begaud B, Moore N, Chaslerie A, Fourrier A, Letenneur L, et al. Benzodiazepine use and risk of dementia: a nested case-control study. J Clin Epidemiol 2002;55:314-318.

31. Wu CS, Wang SC, Chang IS, Lin KM. The association between dementia and long-term use of benzodiazepine in the elderly: nested case-control study using claims data. Am J Geriat Psychiatry 2009;17:614-620.

32. Shash D, Kurth T, Bertrand M, Dufouil C, Barberger-Gateau P, Berr C, et al. Benzodiazepine, psychotropic medication, and dementia: A population-based cohort study. Alzheimers Dement 2016;12:604-613.

33. Baek YH, Lee H, Kim WJ, Chung JE, Pratt N, Kalisch Ellett L, et al. Uncertain Association Between Benzodiazepine Use and the Risk of Dementia: A Cohort Study. J Am Med Dir Assoc 2020;21:201-211.e202.

34. Beydoun MA, Beydoun HA, Gamaldo AA, Teel A, Zonderman AB, Wang Y. Epidemiologic studies of modifiable factors associated with cognition and dementia: systematic review and meta-analysis. BMC Public Health 2014;14:643.

35. Whitmer RA, Gunderson EP, Barrett-Connor E, Quesenberry CP Jr, Yaffe K. Obesity in middle age and future risk of dementia: a 27 year longitudinal population based study. BMJ 2005;330:1360.

36. Cai L, Huang J. Schizophrenia and risk of dementia: a meta-analysis study. Neuropsychiatr Dis Treat 2018;14:2047-2055.

37. Ku BD, Kim SG, Lee JY, Park KH, Shin JH, Kim KK, et al. Clinical practice guideline for dementia by Clinical Research Center for Dementia of South Korea. J Korean Med Assoc 2011;54:861-875.

38. Taragano FE, Allegri RF, Lyketsos C. Mild behavioral impairment: a prodromal stage of dementia. Dement Neuropsychol 2008;2:256-260.

39. Rocca WA, Petersen RC, Knopman DS, Hebert LE, Evans DA, Hall KS, et al. Trends in the incidence and prevalence of Alzheimer's disease, dementia, and cognitive impairment in the United States. Alzheimers Dement 2011;7:80-93. 
Supplementary Table 1. Case definitions using World Health Organization adverse reactions terminology

\begin{tabular}{lcl}
\hline \multicolumn{1}{c}{ Categories } & ARRN & \multicolumn{1}{c}{ Preferred terms } \\
\hline Cognitive decline & 0199 & $\begin{array}{l}\text { Thinking abnormal, bradyphrenia, cerebration impaired, mental concentration difficulty, intellect impaired, } \\
\text { mental torpor, judgment impaired, mental dullness, thinking irrational, thought block, bradypsychic } \\
\text { responses, mental state abnormal, mental state abnormal aggravated, dyslogia, perception disturbance, } \\
\text { tachyphrenia }\end{array}$ \\
& $1877 \quad \begin{array}{l}\text { Cognitive disorders, cognitive function abnormal, cognitive deterioration, acalculia, amusia } \\
\text { Dementia }\end{array}$ & $\begin{array}{ll}\text { Dementia, dementia acquired, mental deterioration, dementia aggravation, Pick's disease, senile dementia, } \\
\text { Korsakoff's psychosis }\end{array}$ \\
& $1453 \quad$ Alzheimer's disease \\
\hline
\end{tabular}

ARRN: Adverse Reaction Record Number 